EUROPEAN JOURNAL OF PURE AND APPLIED MATHEMATICS

Vol. 11, No. 1, 2018, 110-137

ISSN 1307-5543 - www.ejpam.com

Published by New York Business Global

\title{
A new mathematical model of heat equations and its application on the agriculture soil
}

\author{
Ali Allahem ${ }^{1,2}$, Salah Boulaaras ${ }^{2,3, *}$, Khaled Zennir ${ }^{2,4}$ and Mohamed Haiour ${ }^{5}$ \\ 1 Department of Mathematics, College of Sciences, Qassim University, \\ Kingdom of Saudi Arabia \\ 2 Department of Mathematics, College of Sciences and Arts, Al-Rass, Qassim University, \\ Kingdom of Saudi Arabia \\ 3 Laboratory of Fundamental and Applied Mathematics of Oran (LMFAO), University of \\ Oran 1, Ahmed Benbella, Algeria. \\ 4 LAMAHIS Laboratory, Department of Mathematics, University August 20, 1955- Skikda, \\ 21000, Algeria \\ 5 Department of Mathematics, Faculty of Sciences, Annaba University, Algeria
}

\begin{abstract}
The goal of this paper is to provide a mathematical model which based on numerical simulation applied to heat equations and comparing the obtained results with virtual temperatures results for different depths of the homogeneous agricultural soil. The model was treated through this research that enable to know the degree of soil temperature homogeneous without recourse to tools of measuring physical expensive. The importance of this lies in the agricultural seed germination at temperatures suitable.
\end{abstract}

2010 Mathematics Subject Classifications: AMS 65N06, 65N12, 65F05

Key Words and Phrases: Numerical model, Finite elements, Temperature fields, Meteorology,HeatSoil, Agricultural

\section{Introduction}

In the recent years, the alternative methods are used to speed in solving partial differential equations that have been explained the natural phenomena, one of these methods is the finite element method. In this project, we use the finite element method which belong to the family in general methods of Galerkin. These are used instead of the style differences limited (considered simplistic) to resolve the horizontal and vertical fields in numerical model. Galerkin methods can be used in solving systems of partial differential equations fixed and the problems of border free evolutionary (see reference [1], [2], [5],

${ }^{*}$ Corresponding author.

Email addresses: a.allahem@qu.edu.sa (A. Allahem), saleh_boulaares@yahoo.fr or S.Boularas@qu.edu.sa (S. Boulaaras), khaledzennir2@yahoo.com or k.zennir@qu.edu.sa (Kh. Zennir), haiourm@yahoo.fr (M. Haiour) 
[6], [7], [12], [13] and [7]) do not use this method directly in the field of values at grid points after the partition of scale, so as to improve the accuracy of ordinary differential equations systems solutions. There are two methods in this process: the specific elements of the functions of the zero-way, and the way spectral. Finite element method is one of the ways of applied Mathematics. And we put it in space, using the principles inherited from the transformative formulation or weak wording, separate mathematical algorithm to find approximate solution to the problem of free boundary (see [3], [4] and [14]). It is said Dirichlet conditions or Neumann data.

Finite element method differs from the spectral method because it is not exhaustive, but rather to determine local values. However, it is a rough distinct function defined on the whole region and not just separate points (see [8] and [10]).

The techniques used can solve with numerical methods, the equations describing the behavior of the atmosphere [16] and [13], that is to say to determine future values of its parameters characteristics starting from known initial values through meteorological observations [9], [11], [18] and [15]. The use of numerical computation is required to solve these systems of nonlinear equations whose solutions cannot be determined analytically in the general case. The construction of a numerical model of the atmosphere consists of two distinct stages: the first is to establish a system of equations governing the behavior of continuous atmosphere that called the second scan is to replace the equations on continuous variables by equations involving discrete variable whose solutions are obtained by means of a suitable algorithm. The results of a numerical weather prediction, solutions of discretized equations of dynamic meteorology, therefore depend on the adopted scanning. In the late '40s methods of grid points were used to model the universally large-scale atmospheric flow. During recent years ([12], [19] and [20]), alternative methods have been used, one of these methods is the finite element method. We present here the main tools for implementations of the finite element method which belongs to the more general family of Galerkin methods. These are commonly used instead of the finite difference method to treat the horizontal and vertical fields in weather prediction models. Galerkin methods, which can solve numerically systems of partial differential equations, do not directly use the field values at the points of a grid, but use series expansions of functions suitably chosen so to be reduced to the solution of a system of ordinary differential equations. There are two types of methods within this process: the finite element method for which the functions are zero, except for a small area or they are equal to the low-order polynomials, and the spectral method in which the functions are the functions of a spatial operator defined on the whole area of the works.

The finite element method is one of the tools of applied mathematics. It is puts in place, using principles inherited from the variational formulation or weak formulation, a discrete mathematical algorithm for finding an approximate solution of a partial differential equation on a compact domain with boundary conditions and / or in the interior of the compact. It speaks Dirichlet conditions (values at the edges) or Neumann (gradients at the edges). The finite element method is different than the spectral because it is not comprehensive, but rather determined by local values. However, it is distinct approximations gridded because the function is defined over the entire region and not just 
the discrete points. As in the case of other numerical methods, questions arise as to the discretization: Existence and uniqueness of solutions, stability, convergence and of course, the error measure between a discrete and a unique solution of the initial problem.

Our goal in this paper is to highlight the application of the method to an equation of parabolic type. In the practical part, we applied this method to the diffusion equation for virtual temperatures results in the ground in Qassim province in Kingdom of Saudi Arabia.

This paper is organized as follows: In the first part, we introduce some necessary notations of the problem, then we study the numerical analysis side of the heat equations and we obtain the weak formulation of our model using the theta time scheme combined with a finite element method. Then the stability analysis of the proposed schemes and the theoretical side of the full discrete problem is established. In the second part, we present a one-dimensional numerical prediction model that predicts the temperature field for different depths of soil homogeneous and for different times and different days of a given month. By using the finite element approximation of the Lagrange polynomial of order 1 , determining the change in temperature in the soil over time by the knowledge of the parameters and characteristics of the soil. During a period of 24 hours, the temperature surface is substantially the response to radiation received diurnal. Exchanges heat does make the firsts in soil layers that can return to the surface if necessary, part of the energy. By cons, in a day, the heat storage in the soil (thermal inertia) concerns only the first layer of soil not exceeding $60 \mathrm{~cm}$. I.e. the temperature field in the surface layer of the soil, which extends to a depth of $60 \mathrm{~cm}$, is still sensitive to climatic variations recorded on the surface. The objective of this work is precisely to propose a prediction model based on dimensional numerical finite element approximation for the evolution of the temperature field in a homogeneous soil. Finally, we perform virtual measurements of the soil temperature and in the soil, that is to say, we use virtual data to prove the accuracy of the numerical method by using the error estimate.

\section{Temperature diffusion equation}

The objective of this work is precisely to propose a one-dimensional numerical prediction model based on the finite element approximation for the evolution of the temperature field in a homogeneous soil. For the case of a homogeneous and isotropic soil, the thermal balance on a volume element, gives rise according to the depth $z$, to the equation of propagation of the heat with internal production of energy $f$ which is written:

$$
\left\{\begin{array}{l}
u_{t}-D u_{z z}=f \text { in }[a, b] \times\left[0, t_{f}\right], \\
u(t, a)=u_{s}=\alpha, u(t, b)=\beta, \\
u(0, z)=u_{0}(z),
\end{array}\right.
$$


A. Allahem, S.Boulaaras, K. Zennir and M. Haiour / Eur. J. Pure Appl. Math, 11 (1) (2018), 110-137 113 where

$$
D=\frac{\rho . \lambda}{c_{p}},
$$

and

$\rho$ is the density, $\lambda$ is the thermal conductivity and $c_{p}$ is the specific heat.

According to ([17]), the thermal diffusively in the soil is $10^{-6} \mathrm{~m}^{2} / \mathrm{s}$.

\subsection{Discrete problem}

To solve this problem (1), one can proceed in two stages. In a first step, we discretizes in space by finite elements, that is to say that we approach the space $H^{1}$ by a finite dimension space $V_{1}^{h}$ defined as:

$$
V_{1}^{h}=\left\{\begin{array}{c}
v_{h} \in C^{2}(\Omega) \cap H^{1}(\Omega), \\
\left.v_{h}\right|_{I=[a, b]} \in P_{1} \text { in } \Omega \\
\text { and } u(a)=\alpha, u(b)=\beta .
\end{array}\right\}
$$

\subsubsection{Spatial discretization}

We then define the semi-discretized problem, multiplying equation (1) by a test function $v \in$ $H^{1}([a, b])$ and we integrate on $[a, b]=\Omega$, we get

$$
\int_{\Omega} u_{t} v d z-D \int_{\Omega} u_{z z} v d z=\int_{\Omega} f v d z .
$$

To introduce an approximation $u^{h} \in V_{1}^{h} \subset H^{1}(\Omega)$, we will introduce a mesh of $\Omega$ composed of $N+1$ points and if the mesh is uniform, the step of spatial discretization is

$$
h=\frac{b-a}{N} .
$$

But in our case the mesh is not uniform, we take:

$$
h=\max h_{j} .
$$

We introduce the following piecewise linear approximation:

$$
V_{1}^{h}=\left\{\begin{array}{c}
v^{h} \in C^{2}(\Omega) \cap H^{1}(\Omega): \\
v^{h}=p_{1} \in P_{1} \\
\left.\right|_{I j} \\
\text { and } v^{h}(a)=\alpha, v^{h}(b)=0,
\end{array}\right\}
$$


A. Allahem, S.Boulaaras, K. Zennir and M. Haiour / Eur. J. Pure Appl. Math, 11 (1) (2018), 110-137 114 where

$$
v^{h}=\sum_{j=1}^{N-1} v_{j}(z) \varphi_{j}(z), u^{h}=\sum_{i=0}^{N} u_{i}(t) \varphi_{i}(z)
$$

with $\left\{\varphi_{i}\right\}_{i=1, \ldots, N}$ form a vector basis $u^{h}$ and $\left\{\varphi_{j}\right\}_{j=1, \ldots, N}$ for $v^{h}$.

Problem (3) is equivalent to

$$
\begin{aligned}
& \underbrace{\int_{\Omega} u_{t} \cdot v}_{A} d z+D(\underbrace{\int_{\Omega} u_{z z} \cdot v_{z}}_{B} d z \\
& -\underbrace{\int_{\Gamma} u_{z z} \cdot \vec{\eta} \cdot v \cdot d \sigma}_{C})=\int_{\Omega} f v d z .
\end{aligned}
$$

Terms $B$ and $C$ are obtained using Green's formula as:

$$
\begin{aligned}
& \int_{\Omega} u_{z z} v_{z} \cdot v d z=\underbrace{\int_{\Omega} u_{z} v_{z} d z}_{B}-\underbrace{\left.\int_{\Gamma} u_{z} \cdot \eta \cdot v \cdot d \sigma\right)}_{C}
\end{aligned}
$$

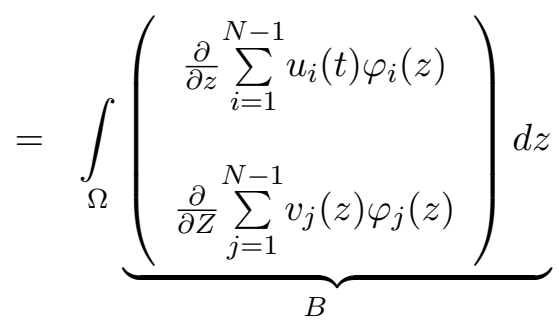

$$
\begin{aligned}
& -\underbrace{\left.\int_{\Gamma} u_{z} \cdot \vec{\eta} \cdot v \cdot d \sigma\right)}_{C}
\end{aligned}
$$

To eliminate the term $C$, we can change the summation of $u$ from $1, \ldots, N-1$ to $0, N$ (that is to say, to consider the boundaries $\alpha$ and $\beta$ ), thus

$$
\int_{\Omega} u_{z z} v d z=\int_{\Omega}\left(\begin{array}{c}
\frac{\partial}{\partial z} \sum_{i=0}^{N} u_{i}(t) \varphi_{i}(z) \\
\frac{\partial}{\partial z} \sum_{j=1}^{N-1} v_{j}(z) \varphi_{j}(z)
\end{array}\right) d z
$$


A. Allahem, S.Boulaaras, K. Zennir and M. Haiour / Eur. J. Pure Appl. Math, 11 (1) (2018), 110-137 115 with

$$
\begin{gathered}
\sum_{i=0}^{N} u_{i}(t) \varphi_{i}(z)=\alpha \varphi_{0}(z) \\
+\sum_{i=1}^{N-1} u_{i}(t) \varphi_{i}(z)+\beta \varphi_{N}(z) .
\end{gathered}
$$

and

$$
A=\frac{\partial}{\partial t}\left(u^{h}, v^{h}\right), B=a\left(u^{h}, v^{h}\right) .
$$

So equation (4) is written in the following variational form:

$$
\frac{\partial}{\partial t}\left(u^{h}, v^{h}\right)+D \cdot a\left(u^{h}, v^{h}\right)=\left(f^{h}, v^{h}\right)
$$

\subsubsection{Time discretization}

In a second step, we discretize (5) with respect to time by using theta scheme. We are therefore looking for a series of elements $u_{n}^{h} \in V^{h}$ which will have to approach $u^{h}\left(t_{n}\right), t_{n}=$ $n \Delta t$ and which are defined by the following formula: find $u_{n}^{h} \in V^{h}$, for all $v^{h} \in V^{h}$ and $\theta \in[0,1]$

$$
\left\{\begin{array}{l}
\left(\frac{u_{n+1}^{h}-u_{n}^{h}}{\Delta t}, v^{h}\right) \\
+D a\left(\theta u_{n+1}^{h}+(1-\theta) u_{n}^{h}, v^{h}\right) \\
=\left(\theta f_{h}^{n+1}+(1-\theta) f_{h}^{n}, v^{h}\right) .
\end{array}\right.
$$

Equation (6) equivalent to

$$
\left\{\begin{array}{l}
\left(u_{n+1}^{h}, v^{h}\right)+D \theta \Delta t . a\left(u_{n+1}^{h}, v^{h}\right) \\
+\left(\theta f_{h}^{n+1}, v^{h}\right)=\left(u_{n}^{h}, v^{h}\right) \\
-D(1-\theta) \Delta t\left(a\left(u_{n}^{h}, v^{h}\right)+\left(f_{h}^{n}, v^{h}\right)\right) .
\end{array}\right.
$$

By recurrence, given $u_{0}^{h}$, we have the existence and uniqueness using Lax-Milgram theorem.

\section{Stability analysis of the theta scheme}

To study the stability, we use the energy increment technique, which allow to conclude without hypothesis on $V^{h}$ when the scheme is unconditionally stable. For the case of a homogeneous and isotropic soil, the thermal balance on a volume element, gives rise according to the depth $z$, to the equation of propagation of the heat without internal 
A. Allahem, S.Boulaaras, K. Zennir and M. Haiour / Eur. J. Pure Appl. Math, 11 (1) (2018), 110-137 116 production of energy (the right hand side of the problem equal zero), thus we can write equation (7) as:

$$
\begin{aligned}
& \left(\frac{u_{n+1}^{h}-u_{n}^{h}}{\Delta t}, v^{h}\right)=D(\theta-1) a\left(u_{n}^{h}, v^{h}\right) \\
& -D \theta a\left(u_{n+1}^{h}, v^{h}\right) \\
& =-D\left[a\left((1-\theta) u_{n}^{h}, v^{h}\right)+a\left(\theta u_{n+1}^{h}, v^{h}\right)\right] \\
& =-D\left[a\left((1-\theta) u_{n}^{h}+\theta u_{n+1}^{h}, v^{h}\right)\right] \\
& =-D\left[a\left(\theta\left(u_{n+1}^{h}-u_{n}^{h}\right)+u_{n}^{h}, v^{h}\right)\right] .
\end{aligned}
$$

Then

$$
\left|\begin{array}{l}
\left(\frac{u_{n+1}^{h}-u_{n}^{h}}{\Delta t}, v^{h}\right) \\
+D\left[a\left(\theta\left(u_{n+1}^{h}-u_{n}^{h}\right)+u_{n}^{h}, v^{h}\right)\right]
\end{array}\right|=0 .
$$

Putting the test function

$$
\begin{aligned}
v^{h} & =\theta\left(u_{n+1}^{h}-u_{n}^{h}\right)+u_{n}^{h} \\
& =\widetilde{u}^{h} .
\end{aligned}
$$

Equation (8) becomes

$$
\begin{aligned}
& \underbrace{\left(u_{n+1}^{h}-u_{n}^{h}, \theta\left(u_{n+1}^{h}-u_{n}^{h}\right)+u_{n}^{h}\right)}_{A} \\
& +\underbrace{D \Delta t a\left(\widetilde{u}^{h}, \widetilde{u}^{h}\right)}_{B}=0 .
\end{aligned}
$$

The term $A$ of the equation equivalent to

$$
\begin{aligned}
& A=\left(u_{n+1}^{h}-u_{n}^{h}, \theta\left(u_{n+1}^{h}-u_{n}^{h}\right)\right) \\
& +\left(u_{n+1}^{h}-u_{n}^{h}, u_{n}^{h}\right) \\
& =\theta\left(u_{n+1}^{h}-u_{n}^{h}, u_{n+1}^{h}-u_{n}^{h}\right) \\
& -\frac{1}{2}\left(u_{n+1}^{h}-u_{n}^{h},-2 u_{n}^{h}\right) .
\end{aligned}
$$


A. Allahem, S.Boulaaras, K. Zennir and M. Haiour / Eur. J. Pure Appl. Math, 11 (1) (2018), 110-137 117 Thus

$$
\begin{aligned}
& A=\theta\left(u_{n+1}^{h}-u_{n}^{h}, u_{n+1}^{h}-u_{n}^{h}\right) \\
& -\frac{1}{2}\left(u_{n+1}^{h}-u_{n}^{h},-u_{n}^{h}-u_{n}^{h}+u_{n+1}^{h}-u_{n+1}^{h}\right) \\
& =\theta\left(u_{n+1}^{h}-u_{n}^{h}, u_{n+1}^{h}-u_{n}^{h}\right) \\
& -\frac{1}{2}\left(u_{n+1}^{h}-u_{n}^{h}, u_{n+1}^{h}-u_{n}^{h}\right) \\
& =\theta\left(u_{n+1}^{h}-u_{n}^{h}, u_{n+1}^{h}-u_{n}^{h}\right) \\
& -\frac{1}{2}\left(u_{n+1}^{h}-u_{n}^{h}, u_{n+1}^{h}-u_{n}^{h}\right),
\end{aligned}
$$

so

$$
\begin{aligned}
& A=\theta\left(u_{n+1}^{h}-u_{n}^{h}, u_{n+1}^{h}-u_{n}^{h}\right) \\
& -\frac{1}{2}\left(u_{n+1}^{h}-u_{n}^{h}, u_{n+1}^{h}-u_{n}^{h}\right) \\
& =\left(\theta-\frac{1}{2}\right)\left(u_{n+1}^{h}-u_{n}^{h}, u_{n+1}^{h}-u_{n}^{h}\right) \\
& +\frac{1}{2}\left(u_{n+1}^{h}-u_{n}^{h}, u_{n+1}^{h}+u_{n}^{h}\right) .
\end{aligned}
$$

Therefore,

$$
\begin{aligned}
& A=\left(\theta-\frac{1}{2}\right)\left\|\left(u_{n+1}^{h}-u_{n}^{h}\right)\right\|_{L^{2}(\Omega)}^{2} \\
& +\frac{1}{2}\left(\int\left(u_{n+1}^{h}\right)^{2}-\left(u_{n}^{h}\right)^{2}\right) \\
& =\left(\theta-\frac{1}{2}\right)\left\|\left(u_{n+1}^{h}-u_{n}^{h}\right)\right\|_{L^{2}(\Omega)}^{2} \\
& +\frac{1}{2}\left\|u_{n+1}^{h}\right\|_{L^{2}(\Omega)}^{2}-\frac{1}{2}\left\|u_{n}^{h}\right\|_{L^{2}(\Omega)}^{2} .
\end{aligned}
$$

By adding the term $A$ into equation (9)

$$
\begin{aligned}
& \left(\theta-\frac{1}{2}\right)\left\|\left(u_{n+1}^{h}-u_{n}^{h}\right)\right\|_{L^{2}(\Omega)}^{2} \\
& +\frac{1}{2}\left\|u_{n+1}^{h}\right\|_{L^{2}(\Omega)}^{2} \\
& -\frac{1}{2}\left\|u_{n}^{h}\right\|_{L^{2}(\Omega)}^{2}+D \Delta t a(\widetilde{u}, \widetilde{u})=0 .
\end{aligned}
$$

This means 
A. Allahem, S.Boulaaras, K. Zennir and M. Haiour / Eur. J. Pure Appl. Math, 11 (1) (2018), 110-137 118

$$
\begin{aligned}
& \frac{1}{2}\left\|u_{n+1}^{h}\right\|_{L^{2}(\Omega)}^{2}-\frac{1}{2}\left\|u_{n}^{h}\right\|_{L^{2}(\Omega)}^{2} \\
& +\Delta t D a(\widetilde{u}, \widetilde{u}) \\
& =-\left(\theta-\frac{1}{2}\right)\left\|\left(u_{n+1}^{h}-u_{n}^{h}\right)\right\|_{L^{2}(\Omega)}^{2} .
\end{aligned}
$$

If $\theta \geq \frac{1}{2}$, we have

$$
\begin{aligned}
& \frac{1}{2}\left\|u_{n+1}^{h}\right\|_{L^{2}(\Omega)}^{2}-\frac{1}{2}\left\|u_{n}^{h}\right\|_{L^{2}(\Omega)}^{2} \\
& +\Delta t D a\left(\widetilde{u}^{h}, \widetilde{u}^{h}\right) \leq 0
\end{aligned}
$$

thus

$$
\begin{aligned}
& \left\|u_{n+1}^{h}\right\|_{L^{2}(\Omega)}^{2}+2 \Delta t D a\left(\widetilde{u}^{h}, \widetilde{u}^{h}\right) \\
& \leq\left\|u_{n}^{h}\right\|_{L^{2}(\Omega)}^{2}
\end{aligned}
$$

then

$$
\begin{aligned}
& \left\|u_{n+1}^{h}\right\|_{L^{2}(\Omega)}^{2}+2 \Delta t D\left\|\frac{\partial \widetilde{u}^{h}}{\partial z}\right\|_{L^{2}(\Omega)}^{2} \\
& \leq\left\|u_{n}^{h}\right\|_{L^{2}(\Omega)}^{2} .
\end{aligned}
$$

By summation with respect to $n$, we find

$$
\begin{aligned}
& \left\|u_{n}^{h}\right\|_{L^{2}(\Omega)}^{2}+2 \Delta t D \sum_{n=1}^{n}\left\|\frac{\partial \widetilde{u}^{h}}{\partial z}\right\|_{L^{2}(\Omega)}^{2} \\
& \leq \underbrace{\left\|u_{0}^{h}\right\|_{L^{2}(\Omega)}^{2}}_{\text {known }} .
\end{aligned}
$$

It is deduced that for $\theta \geq \frac{1}{2}$ the scheme is unconditionally stable.

\subsection{Matrix formulation of the problem}

We can reformulate equation $(7)$

$$
\begin{aligned}
& \left(u_{n+1}^{h}, v^{h}\right)+D \theta \Delta t a\left(u_{n+1}^{h}, v^{h}\right) \\
& =\left(u_{n}^{h}, v^{h}\right)-D(1-\theta) \Delta t a\left(u_{n}^{h}, v^{h}\right), \\
& \omega=\theta \Delta t D .
\end{aligned}
$$


A. Allahem, S.Boulaaras, K. Zennir and M. Haiour / Eur. J. Pure Appl. Math, 11 (1) (2018), 110-137 119 We have again

$$
\begin{aligned}
& \int_{\Omega}\left(\sum_{i=1}^{N-1} u_{i}^{n+1}(t) \varphi_{i}(z) \sum_{j=1}^{n-1} v_{j} \varphi_{j}(z)\right) d z \\
& +\omega \int_{\Omega} \frac{\partial}{\partial z}\left(\sum_{i=1}^{N-1} u_{i}^{n+1}(t) \varphi_{i}(z)\right) \frac{\partial}{\partial z}\left(\sum_{j=1}^{n-1} v_{j} \varphi_{j}(z)\right) d z \\
& =\int_{\Omega}\left(\sum_{i=1}^{N-1} u_{i}^{n}(t) \varphi_{i}(z) \sum_{j=1}^{n-1} v_{j} \varphi_{j}(z)\right) d z \\
& -(1-\theta) \Delta t D \int_{\Omega} \frac{\partial}{\partial z}\left(\sum_{i=0}^{N} u_{i}^{n}(t) \varphi_{i}(z)\right) \frac{\partial}{\partial z}\left(\sum_{j=1}^{n-1} v_{j} \varphi_{j}(z)\right) d z .
\end{aligned}
$$

Equation (11) is equal to

$$
\begin{aligned}
& \sum_{i=1}^{N-1} u_{i}^{n+1}(t) \int_{\sup p \varphi_{i} \cap \sup p \varphi_{j}} \varphi_{i}(z) \varphi_{j}(z) d z \\
& +\omega \sum_{i=1}^{N-1} u_{i}^{n+1}(t) \int_{\sup p \varphi_{i} \cap \sup p \varphi_{j}} \frac{\partial}{\partial z} \varphi_{i}(z) \frac{\partial}{\partial z} \varphi_{j}(z) d z \\
& =\sum_{i=1}^{N-1} u_{i}^{n}(t) \int_{\sup p \varphi_{i} \cap \sup p \varphi_{j}} \varphi_{i}(z) \varphi_{j}(z) d z \\
& -(1-\theta) \Delta t D \sum_{i=0}^{N} u_{i}^{n}(t) \int_{\sup p \varphi_{i} \cap \sup p \varphi_{j}} \frac{\partial}{\partial z} \varphi_{i}(z) \frac{\partial}{\partial z} \varphi_{j}(z) d z,
\end{aligned}
$$

thus 
A. Allahem, S.Boulaaras, K. Zennir and M. Haiour / Eur. J. Pure Appl. Math, 11 (1) (2018), 110-137 120

$$
\begin{aligned}
& \sum_{i=1}^{N-1} u_{i}^{n+1}(t)\left(\begin{array}{c}
\int_{\operatorname{supp} \varphi_{i} \cap \operatorname{supp} \varphi_{j}} \varphi_{i}(z) \cdot \varphi_{j}(z) d Z \\
+\omega \int_{\sup p \varphi_{i} \cap \sup p \varphi_{j}} \frac{\partial}{\partial Z} \varphi_{i}(z) \frac{\partial}{\partial Z} \varphi_{j}(z) d z
\end{array}\right) \\
& =-\alpha(1-\theta) \Delta t D \int_{\sup p \varphi_{i} \cap \sup p \varphi_{j}} \frac{\partial}{\partial z} \varphi_{0}(z) \frac{\partial}{\partial z} \varphi_{1}(z) d z \\
& -\beta(1-\theta) \Delta t D \int_{\sup p \varphi_{i} \cap \sup p \varphi_{j}} \frac{\partial}{\partial z} \varphi_{N}(z) \frac{\partial}{\partial z} \varphi_{N-1}(z) d z \\
& +\sum_{i=1}^{N-1} u_{i}^{n}(t)\left(\begin{array}{c}
\int_{\sup p \varphi_{i} \cap \sup p \varphi_{j}} \varphi_{i}(z) \cdot \varphi_{j}(z) d x \\
-(1-\theta) \Delta t D \int_{\sup p \varphi_{i} \cap \sup p \varphi_{j}} \frac{\partial}{\partial z} \varphi_{i}(x) \frac{\partial}{\partial z} \varphi_{j}(z) d z .
\end{array}\right)
\end{aligned}
$$

Then

$$
\begin{aligned}
& \sum_{i=1}^{N-1} u_{i}^{n+1}(t)\left(\begin{array}{c}
\int_{x_{i-1}}^{x_{i+1}} \varphi_{i}(z) \cdot \varphi_{j}(z) d x \\
+\theta \Delta t D \int_{x_{i-1}}^{x_{i+1}} \frac{\partial}{\partial z} \varphi_{i}(z) \cdot \frac{\partial}{\partial z} \varphi_{j}(z) d z
\end{array}\right) \\
& =-\alpha(1-\theta) \Delta t D \cdot \int_{\sup p \varphi_{i} \cap \sup p \varphi_{j}} \frac{\partial}{\partial z} \varphi_{0}(z) \cdot \frac{\partial}{\partial z} \varphi_{1}(z) d z \\
& -\beta(1-\theta) \Delta t D \cdot \int_{\sup p \varphi_{i} \cap \sup p \varphi_{j}} \frac{\partial}{\partial z} \varphi_{N}(z) \frac{\partial}{\partial z} \varphi_{N-1}(z) d z \\
& +\sum_{i=1}^{N-1} u_{i}^{n}(t)\left(\begin{array}{c}
\int_{x_{i-1}}^{x_{i+1}} \varphi_{i}(z) \varphi_{j}(z) d z \\
-(1-\theta) \Delta t D \int_{x_{i-1}}^{x_{i+1}} \frac{\partial}{\partial z} \varphi_{i}(z) \frac{\partial}{\partial x} \varphi_{j}(z) d z
\end{array}\right)
\end{aligned}
$$

with basic functions

$$
\varphi_{i}(z)=\left\{\begin{array}{l}
\frac{z-z_{i-1}}{h}, z \in\left[z_{i-1}, z_{i}\right], \\
\frac{Z_{i+1}-Z}{h}, z \in\left[z_{i}, z_{i+1}\right]
\end{array}\right.
$$

and 
A. Allahem, S.Boulaaras, K. Zennir and M. Haiour / Eur. J. Pure Appl. Math, 11 (1) (2018), 110-137 121

$$
\varphi_{i-1}(z)=\left\{\begin{array}{l}
\frac{z-z_{i-2}}{h}, z \in\left[z_{i-2}, z_{i-1}\right] \\
\frac{z_{i}-z}{h}, z \in\left[z_{i-1}, z_{i}\right]
\end{array}\right.
$$

with

$$
h=\left(z_{i+1}-z_{i}\right)=\left(z_{i}-z_{i-1}\right) .
$$

To make the calculations easier, we make a change change such as

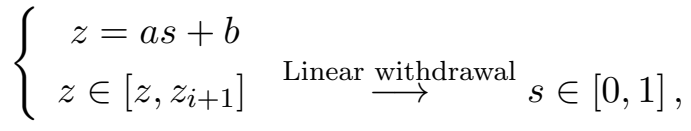

thus, it can be easily found:

$$
\begin{gathered}
\varphi_{0}(s)=\left\{\begin{array}{c}
(1+s), s \in[-1,0], \\
(1-s), s \in[0,1],
\end{array}\right. \\
\varphi_{-1}(s)=\left\{\begin{array}{l}
(1+s), s \in[-2,-1], \\
-s, s \in[-1,0]
\end{array}\right.
\end{gathered}
$$

and

$$
\varphi_{1}(s)=\left\{\begin{array}{l}
(1-s), s \in[0,1] \\
-s, s \in[1,2]
\end{array}\right.
$$

Equation (13) becomes 
A. Allahem, S.Boulaaras, K. Zennir and M. Haiour / Eur. J. Pure Appl. Math, 11 (1) (2018), 110-137 122

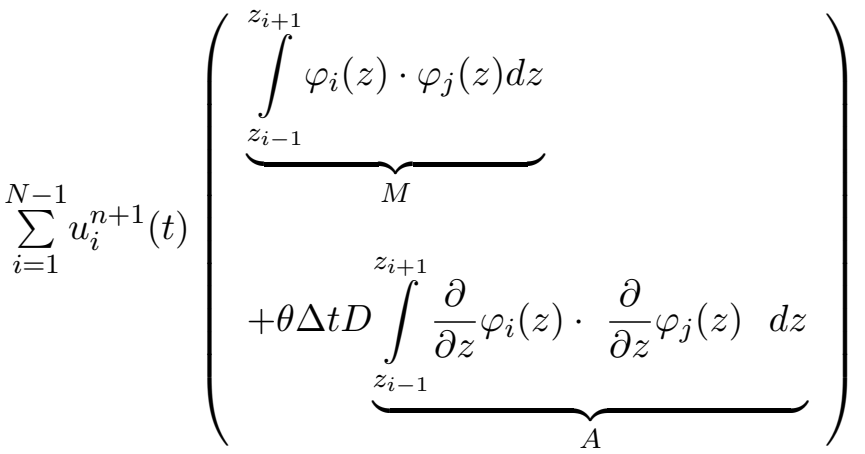

$$
\begin{aligned}
& =-\alpha(1-\theta) \Delta t D \int_{\sup p \varphi_{i} \cap \sup p \varphi_{j}} \frac{\partial}{\partial z} \varphi_{0}(z) \cdot \frac{\partial}{\partial z} \varphi_{1}(z) d z \\
& -\beta(1-\theta) \Delta t D \int_{\sup p \varphi_{i} \cap \sup p \varphi_{j}} \frac{\partial}{\partial z} \varphi_{N}(z) \cdot \frac{\partial}{\partial z} \varphi_{N-1}(z) d z \\
& +\sum_{i=1}^{N-1} u_{i}^{n}(t)\left(\begin{array}{l}
\int_{z_{i-1}}^{z_{i+1}} \varphi_{i}(z) \cdot \varphi_{j}(z) d z \\
-(1-\theta) \Delta t D \int_{z_{i-1}}^{z_{i+1}} \frac{\partial}{\partial Z} \varphi_{i}(z) \cdot \frac{\partial}{\partial z} \varphi_{j}(z) d z .
\end{array}\right)
\end{aligned}
$$

Therefore, we get the following linear system

$$
\begin{aligned}
& (M+\theta \Delta t D A) u^{n+1}=(M-(1-\theta) \Delta t D A) u^{n} \\
& -(1-\theta) \Delta t D B
\end{aligned}
$$

where $M$ and $A$ are symmetric tridiagonal matrix, their dimension is $(N-1) \times(N-1)$ and

$$
\begin{aligned}
& M=m\left(\varphi_{i}, \varphi_{j}\right)=\int_{z_{i-1}}^{z_{i+1}} \varphi_{i}(z) \cdot \varphi_{j}(z) d z \\
& A=a\left(\varphi_{i}, \varphi_{j}\right)=\int_{z_{i-1}}^{z_{i+1}} \varphi_{i}(z) \cdot \varphi_{j}(z) d z
\end{aligned}
$$

with

$$
B=\left(\alpha a\left(\varphi_{0}, \varphi_{1}\right), \ldots, \beta a\left(\varphi_{N}, \varphi_{N-1}\right)\right)^{t} .
$$


In addition, we have $u^{n+1}=\sum_{i=1}^{N-1} u_{i}^{n+1}(t)$ and $u^{n}=\sum_{i=1}^{N-1} T_{i}^{n}(t)$. Now, it is easy to calculate $M\left(\varphi_{i}, \varphi_{j}\right), A\left(\varphi_{i}, \varphi_{j}\right)$ as following:

$$
M\left(\varphi_{i}, \varphi_{j}\right)=\left\{\begin{array}{l}
\frac{2 h}{3}, i=j, \\
\frac{h}{6},|i-j|=1
\end{array}\right.
$$

and

$$
A\left(\varphi_{i}, \varphi_{j}\right)=\left\{\begin{array}{l}
\frac{2}{h}, i=j, \\
-\frac{1}{h},|i-j|=1 .
\end{array}\right.
$$

\section{Application}

\subsection{Error evaluation}

To put our work into practice, measurements of the temperature on the ground and in the soil are made, that is, virtual data was used to highlight the accuracy of the method using the error assessment according to weather temperatures data at Qassim province, Qassim province is one of the thirteen administrative regions of Kingdom of Saudi Arabia. Located at the heart of the country, and almost in the center of the Arabian Peninsula, it has a population of 1,370,727 and an area of $58,046 \mathrm{~km}^{2}$. It is known to be the "alimental basket" of the country, for its agricultural assets.

\subsection{Obtaining initial data}

1-The data are considered according to the weather data: the temperature at agricultural ground level, the maximum and minimum temperature on the ground, the temperatures of four levels in the soil $(10,20,30$ and $50 \mathrm{~cm})$. We consider the data of March 2017 whose selected days are from 7 to 10 .

2-As with all numerical forecast models, data at the boundaries of the domain is needed. The boundary $\alpha$ is the ground temperature that is calculated every 10 minutes (using a linear interpolation between the minimum and the maximum of a given day, while considering the maximum and minimum tim) and the boundary $\beta$ is considered according to a previous study in [17], during a day, the storage of heat in the soil concerns only the first layer of soil not exceeding $60 \mathrm{~cm}$. The results obtained show that the temperature field in the upper soil layer, which extends over a depth of $60 \mathrm{~cm}$, remains sensitive to climatic variations recorded at the surface.

Since the data are not available at $60 \mathrm{~cm}$ in the soil, the temperature was estimated at $60 \mathrm{~cm}$ according to the variation of the thermal gradient observed in the soil. Moreover, after this temperature has been fixed during the whole experiment (this hypothesis is proved by a previous study, the results are in Fig 1). 
Note that the temperature at $60 \mathrm{~cm}$ remains virtuality constant during the 24 hours. 3-Taking $\Delta t=10 \mathrm{~min}$ and $h=\max h_{j}=20 \mathrm{~cm}$.

4-We can turn the model every 10 minutes until while displaying every 12 hours the results obtained and compared with the actual data to evaluate the mean squared error.

$$
E Q M=\sum_{n=1}^{n=N} \sqrt{\frac{1}{N}\left(T_{\text {expected }}-T_{\text {real }}\right)^{2}}
$$

6- The calculations can be done with two methods: the first with initialization, where we can turn the model and initialize the data at $6 \mathrm{~h}, 12 \mathrm{~h}$ and $18 \mathrm{~h}$ and the second is without initialization: the model is rotated during the entire deadline without resetting the data.

\subsection{Virtual experience}

The code used in this work is based on the finite element technique to predict the ground temperature at different levels and for different integration deadlines. For that, we realized two simulations. The first is to start the calculation without initialization and retrieve the forecasts at different times, while for the second, the calculation is done with initialization every twelve hours. We compared the distribution of observed and simulated temperatures. We have also calculated for each deadline the quadratic error and then the mean squared error for the whole simulation and the results of the two simulations are as follows:

\subsubsection{Without initialization}

According to the results of Fig 2, we note that the model is in good agreement with the observations for shallow depths for which it gives fairly virtual temperatures, but differences despite which are weak begin to appear when the depth increases, it is reported that the model returns exactly the same temperature for the depth of $25 \mathrm{~cm}$.

According to the results of Fig 3, we note that the model always starts with a very good behavior for the shallow depths and is able to calculate the same temperature as that measured for an interval between 25 and $30 \mathrm{~cm}$. According to the results in the figures 2 until 8 , we note for deadlines that are too high, namely 36 hours, 48 hours and 60 hours, we notice that there is not a very good agreement between the expected temperatures and those observed for the different depths. The figures show that the model slightly overestimates the temperature for shallow depths and underestimates them for significant depths. It arrives at a good forecast for an average depth between 25 and $30 \mathrm{~cm}$, but remains close to observations and for 72 hours, 84 hours and 96 hours, the comparison between the results of the simulation and the observed data shows that the temperatures are not very well reproduced but remains close to the measurements in the limit where the difference between the two temperatures do not exceed $0.6^{\circ} \mathrm{C}$.

According to the results of Fig 9, from a statistical point of view, the calculation of the quadratic error allows a more detailed view of the behavior of the model. It is clear that the smallest errors were observed for the lowest deadlines this is logical as the model 
starts to move away from the value when the maturities increase, but for our case we notice that the model returns at a fairly good estimate for the deadlines of 84, 96 and 108 hours. We note that the lowest error (0.19) corresponds to the best behavior of the model at the expiry of 24 hours.

\subsubsection{With initialization}

According the results of the 12-hours (see Fig 12) simulation with initialization do not show significant differences between the two simulations, where we find the same behavior of the model with a slight over estimation for shallow depths and a slight underestimate for deeper depths. This can be verified by the value of the squared error which gives exactly the same value for both simulations.

The figures results for the $24 \mathrm{~h}$ and $36 \mathrm{~h}$ (see Fig 13 and Fig 14) maturities allow the results of the simulation to be compared to the measurements taken. If the model starts with a very good behavior for the $24 \mathrm{~h}$ deadline, it moves away slightly from the observations thereafter. A distance materialized by a slight overestimation of the temperature. This does not say that the model diverges completely because the differences are quite small. The opposite is observed for the 36 hour deadline for which the model starts an over-estimation and ends with a good behavior. For the deadlines 96 and $108 \mathrm{~h}$ (see Fig 15 up to Fig 20), we notice that the model is in good agreement with the observations for the important depths for which it gives quite virtual temperatures, but differences despite which are weak not exceeding $0.6 \mathrm{~cm}$, begin to appear when the depth decreases for the 96 hour deadline. An unverified aspect for 108 hours of expiry where the model has an underestimation of the temperature for different depths. It is reported that the statistical results show a positive evolution between the two simulations by the comparison of the mean squared error which goes from 0.46 for the simulation without initialization to 0.34 for the simulation with initialization. However, Note that there is not a very good agreement between the expected temperatures and those observed for different depths. The figure shows that the model slightly overestimates the temperature for the weak and the deep depths and arrives at a good prediction for a depth of $10 \mathrm{~cm}$, but remains close to observations. However, the results of the simulation do not indicate a fairly clear behavior of the model with respect to deadlines and depths. In some cases, there is an overestimation. in other cases an underestimation and a good reproduction in the remaining cases. Although the terms underestimation and overestimation are used, the general behavior remains fairly virtual because of the small difference between measurement and calculation.

\section{Conclusion}

The study showed that the finite element method satisfactorily reflects the variation of temperature fields at different depths of the soil. According to the results obtained, the method gives a good simulation and explains to a precision that does not exceed 0.6 degree maximum, that the model did not diverge even after 108 hours. Logically, the model will 
start to move away from the measure as maturities increase, but this is not the case for our present model and this is due to the assumptions we made: Linear interpolation between minimum and maximum ground temperature to obtain temperatures every 10 minutes; the presence of clouds and dew each morning during the entire deadline, the presence of rains, the presence of plants. We noticed after the simulations that the finite element method estimates with good accuracy the temperature in the soil. The results are quite conclusive as well with an initialization as without. On the other hand, we would like to point out that the results of the simulation could be better if the measurements were made under a greenhouse and thus protected from the hydrometeor which clearly influence the variation of the surface temperature.

\section{Acknowledgements}

The first and second authors gratefully acknowledge Qassim University, represented by the Deanship of Scientific Research, on the material support for this research under number (1460-alrasscac-2016-1-12-S) during the academic year 1437 AH/ 2016 AD. Also the authors would like to thank the anonymous referees and the handling editor for their careful reading and for relevant remarks/suggestions which helped them to improve the paper.

\section{References}

[1] M. Ainsworth, Dispersive and dissipative behavior of high order Discontinuous Galerkin finite element methods. Journal of Computational Physics, 198(1):106-130, 2004 .

[2] A. Arakawa, V.R. Lamb, Computational design of the basic dynamical processes of the UCLA general circulation model Methods Comput. Phys., 17:173-265.

[3] S. Boulaaras and M. Haiour. " $L^{\infty}$-asymptotic behavior for a finite element approximation in parabolic quasi-variational inequalities related to impulse control problem", Applied Mathematics and Computation, Vol. 217, No. 3, 2011, pp. 6443-6450,

[4] 15- S. Boulaaras, M. Haiour: A General Case for the Maximum Norm Analysis of an Overlapping Schwarz Methods of Evolutionary HJB Equation with Nonlinear Source Terms with the Mixed Boundary Conditions, Applied Mathematics \& Information Sciences 9(3): (2015)1247-1257 .

[5] J.H. Atkinson, J.J. Westerink, R.A. Luettich, Two dimensional dispersion analyses of finite element approximations to the shallow water equations International Journal for Numerical Methods in Fluids, 45:715-749, 2004.

[6] M.L. Batteen, Y.-J. Han, On the computational noise of finite-difference schemes used in ocean models Tellus, 33:387-396, 1981. 
[7] J.-M. Beckers and E. Deleersnijder, Stability of a fbtcs scheme applied to the propagation of shallow-water inertia-gravity waves on various space grids. Journal of Computational Physics, 108:95-104, 1993.

[8] P.-E. Bernard, J.-F. Remacle, V. Legat and E. Deleersnijder, Dispersion analysis of Discontinuous Galerkin Schemes applied to Poincaré, Kelvin and Rossby waves Journal of Scientific Computing, 34:26-47.

[9] B. Cushman-Roisin, Introduction to Geophysical Fluid Dynamics Prentice-Hall, 1994.

[10] P.G. Ciarlet, Introduction à l'analyse numérique matricielle et à l'optimisation Masson, paris, 1990.

[11] J.K. Dukowicz Mesh effects for Rossby waves Journal of Computational Physics, 119: 188-194, 1995.

[12] R. Glowinski, Numerecal methods for Nolinear variational problems. Springer -Verlag, 1982.

[13] G. Guyot, Cours de Bioclimatologie, Chapitre II : Echanges de Chaleur et de Masse par Conduction et Convection', INRA Bioclimatologie, BP. 91, Montfavet cedex, 1992.

[14] M. Boulbrachene, M. Haiour, The finite element approximation of Hamilton Jacobi Bellman equations, Comput. Math. Appl. (2001) 41, 993-1007.

[15] R. Haxaire, 'Caractérisation et Modélisation des Ecoulements d'Air dans une Serre ', Thèse de Doctorat de l'Université de Nice, France, 1999.

[16] A. Majda. Introduction to PDE's and waves for the atmosphere and ocean . American Mathematical Society, 2003.

[17] R.Nebbali et S. Makhlouf, Dtermination de la distribution du champ de tempratures dans le sol, par un modle semi-analytique.Conditions aux limites pour les besoins de simulation d.une serre de culture, Universit Mouloud Mammeri, 15000 Tizi-Ouzou, Algrie. 2000.

[18] D.A. Randall Geostrophic Adjustment and the Finite-Difference Shallow-Water Equations Monthly Weather Review, 122:1371-1377, 1994.

[19] V. Rostand, D.Y. Le Roux Raviart-Thomas and Brezzi-Douglas-Marini finite element approximations of the shallow water equations International Journal for Numerical Methods in Fluids, (published online), DOI:10.1002/fld.1668, 2007.

[20] L. White and E. Deleersnijder. Diagnoses of vertical transport in a three-dimensional finiteelement model of the tidal circulation around an island. Estuarine Coastal and Shelf Science, 74:655-669, 2007. 
REFERENCES

\section{ANNEX}

\subsection{Figures of without initialization results}

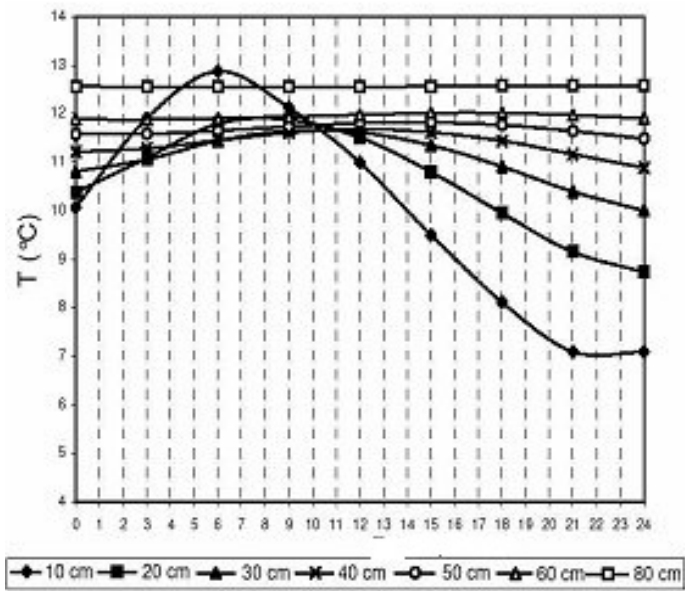

Figure 1: Temperature evolution in the soil for different depths.

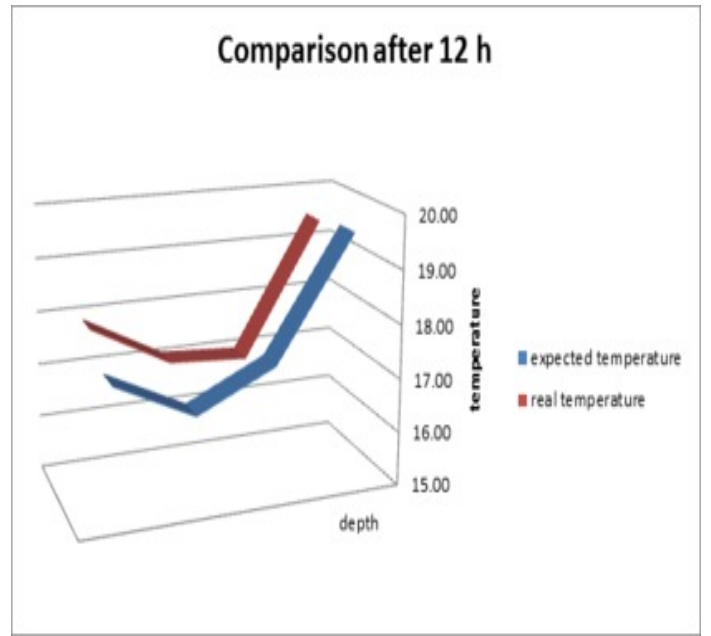

Figure 2: Comparison after $12 \mathrm{~h}$.

\subsection{Figures of with initialization results}




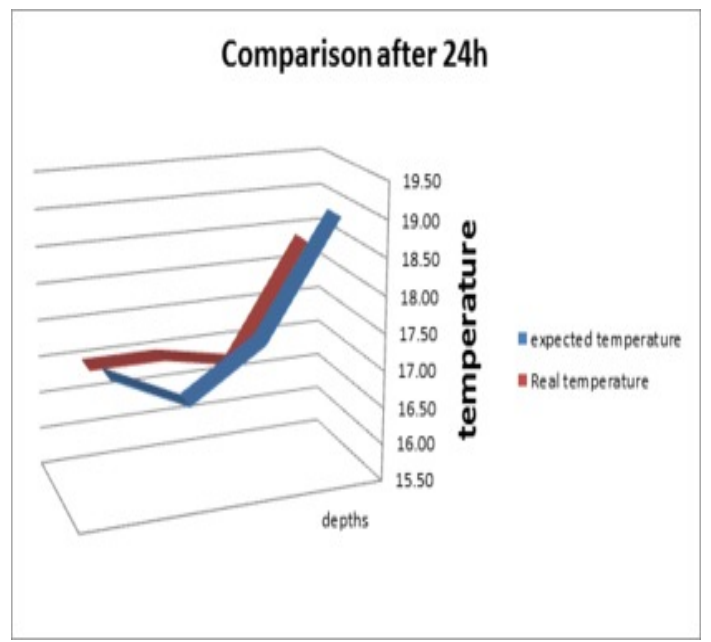

Figure 3: Comparison after 24h.

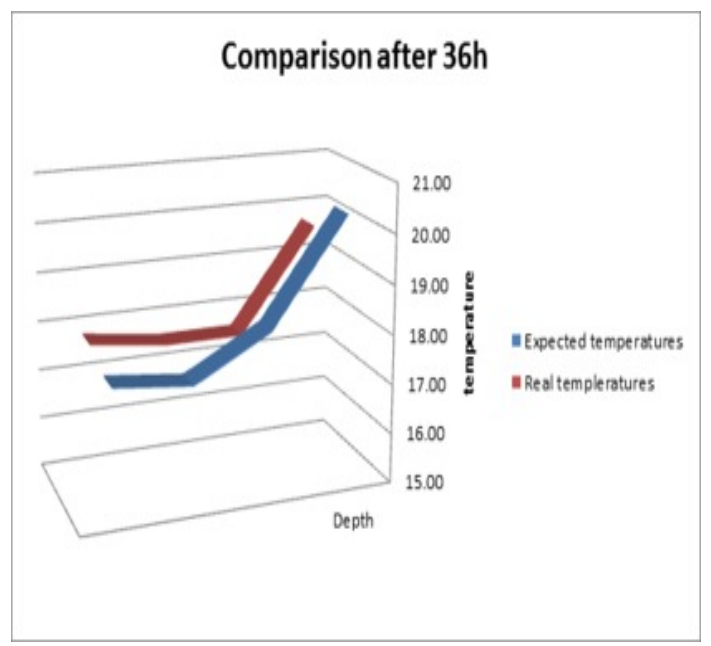

Figure 4: Comparison after 36h. 


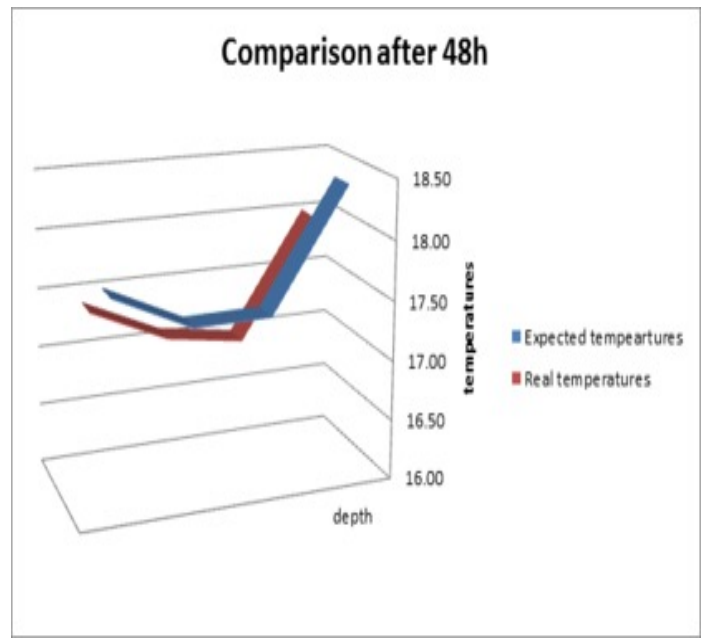

Figure 5: Comparison after 48h.

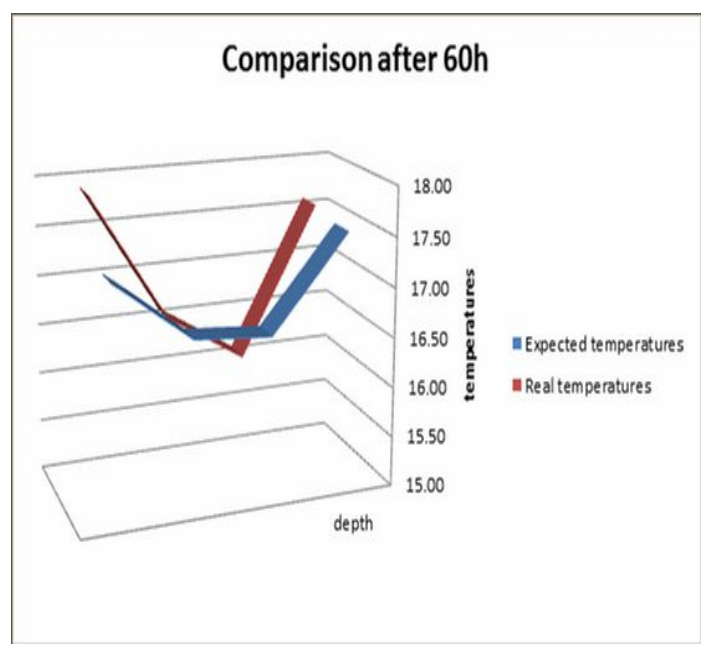

Figure 6: Comparison after 60h. 


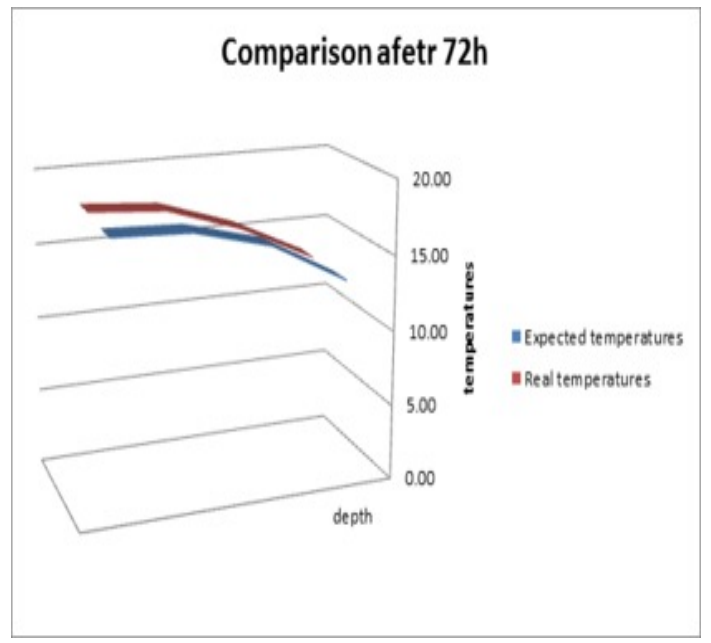

Figure 7: Comparison after 72h.

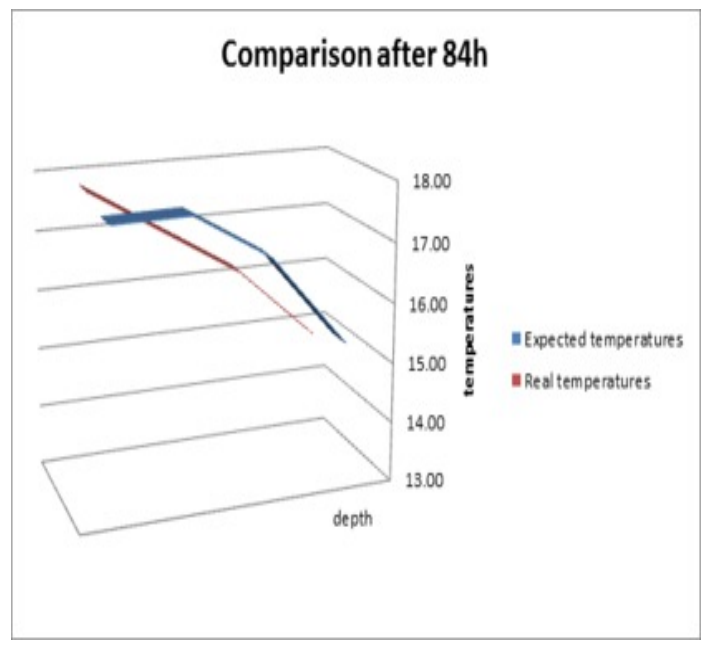

Figure 8: Comparison after 84h. 


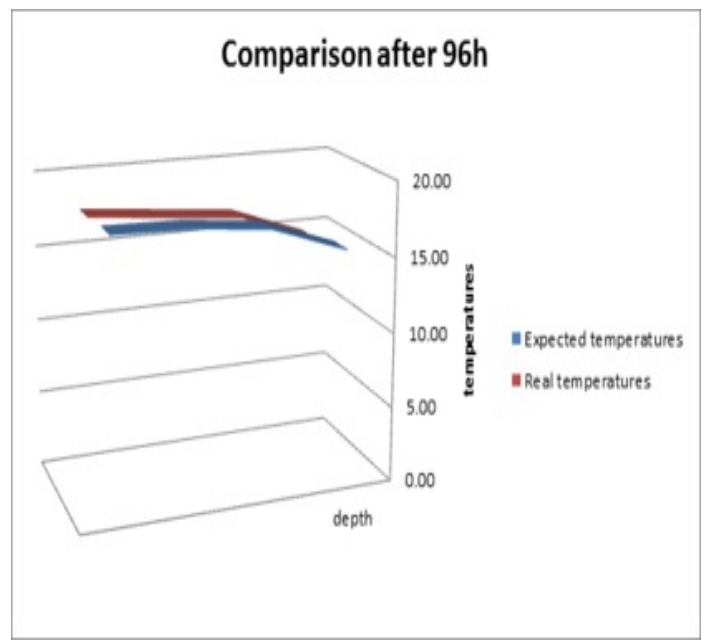

Figure 9: Comparison after 96h.

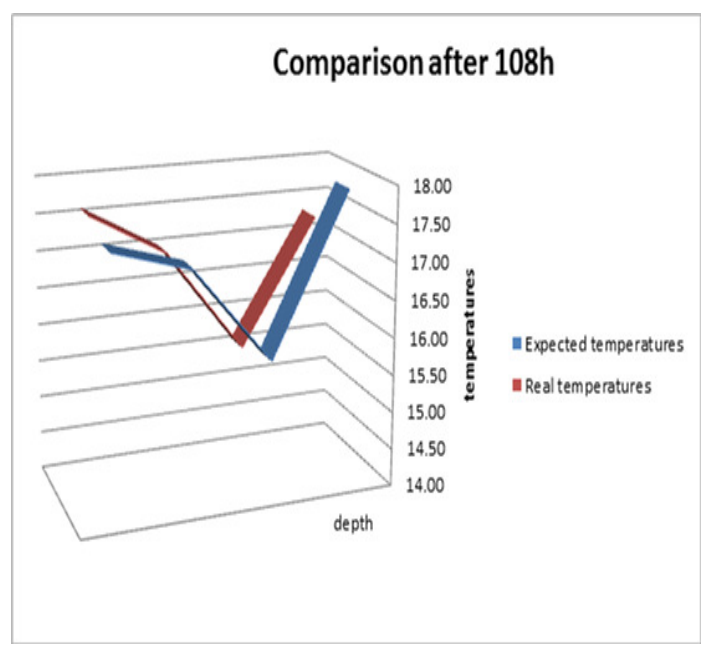

Figure 10: Comparison after $108 \mathrm{~h}$. 


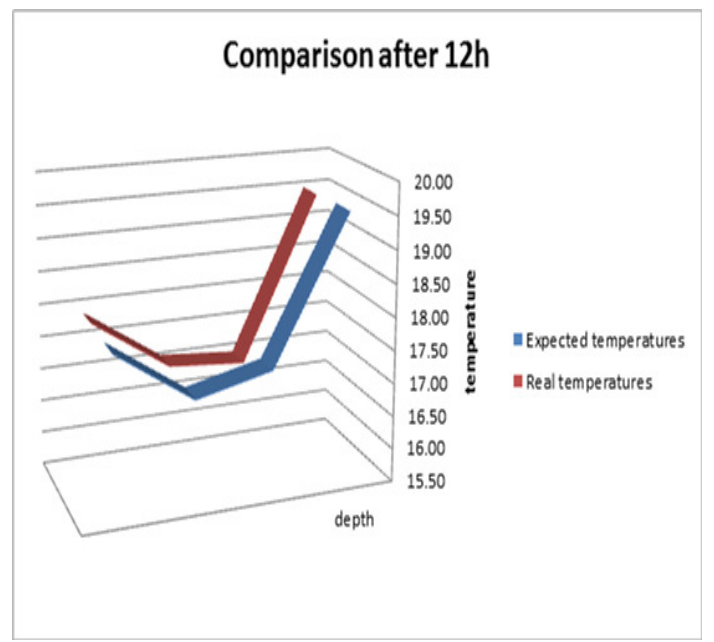

Figure 11: Comparison after $12 \mathrm{~h}$.

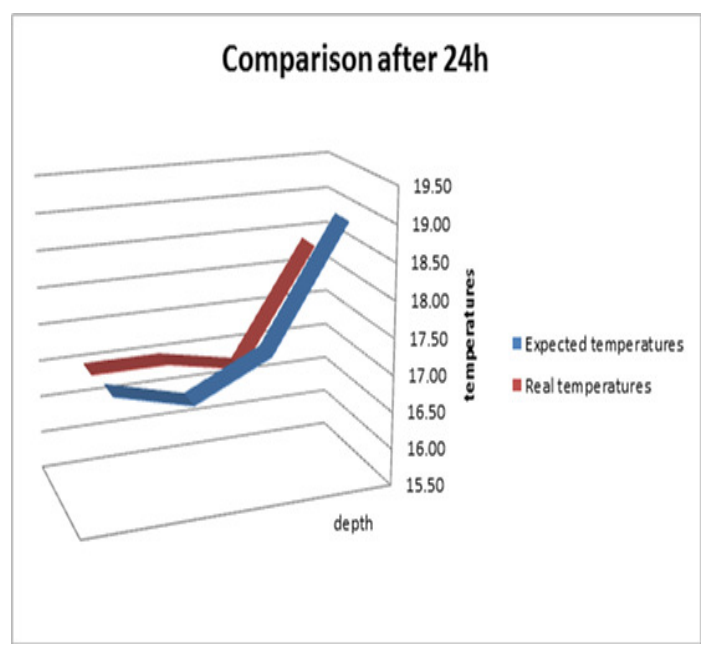

Figure 12: Comparison after 24h. 


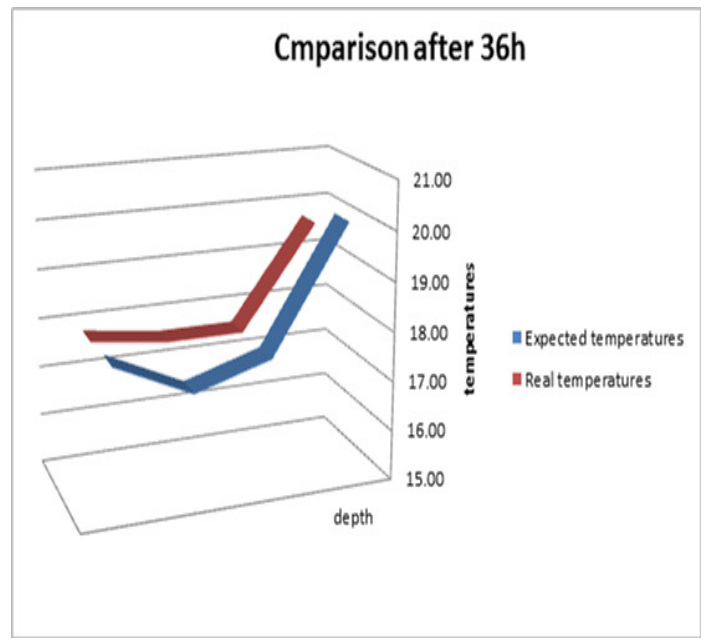

Figure 13: Comparison after 36h.

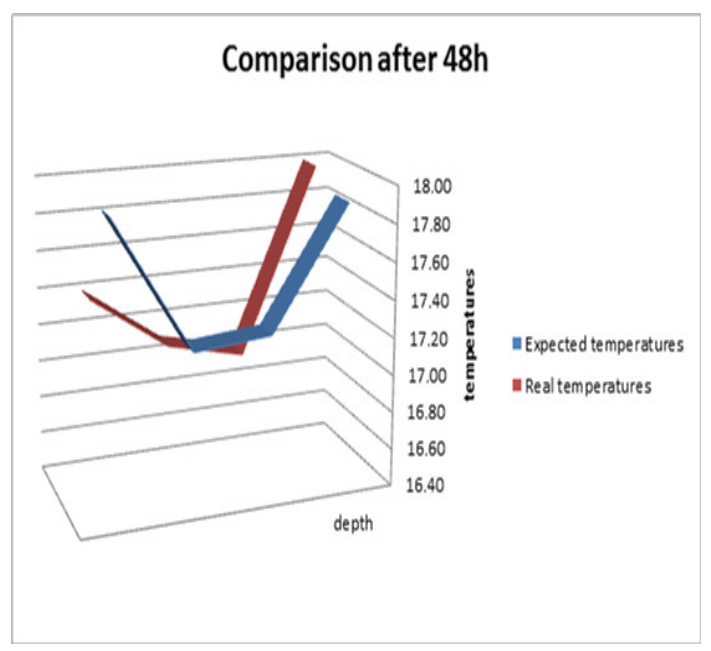

Figure 14: Comparison after 48h. 


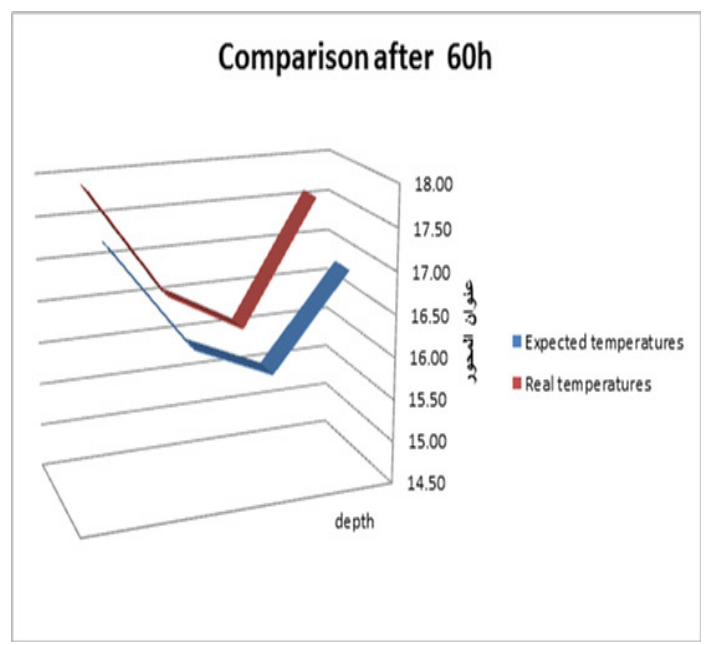

Figure 15: Comparison after 60h.

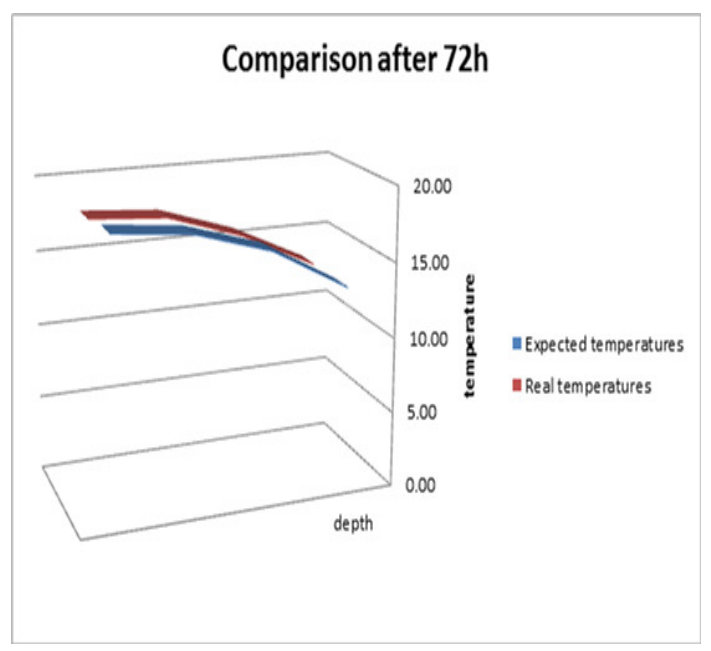

Figure 16: Comparison after $72 \mathrm{~h}$. 


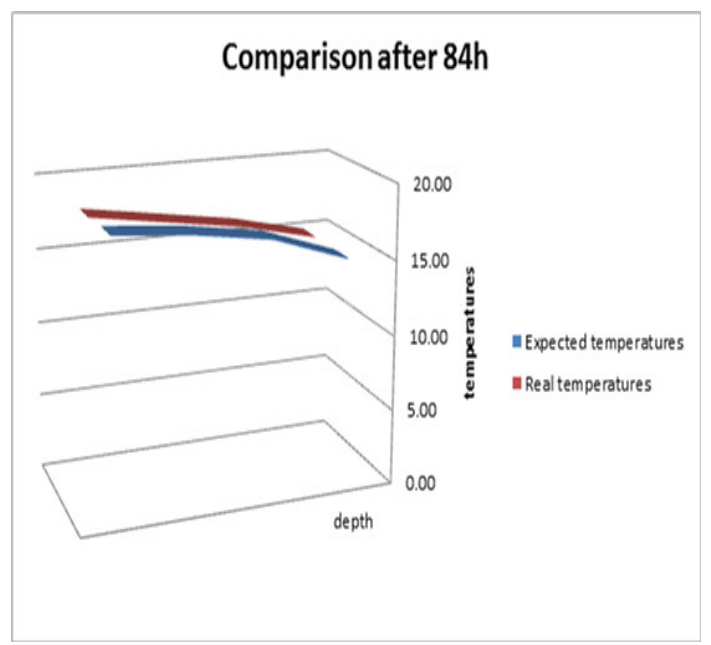

Figure 17: Comparison after 84h.

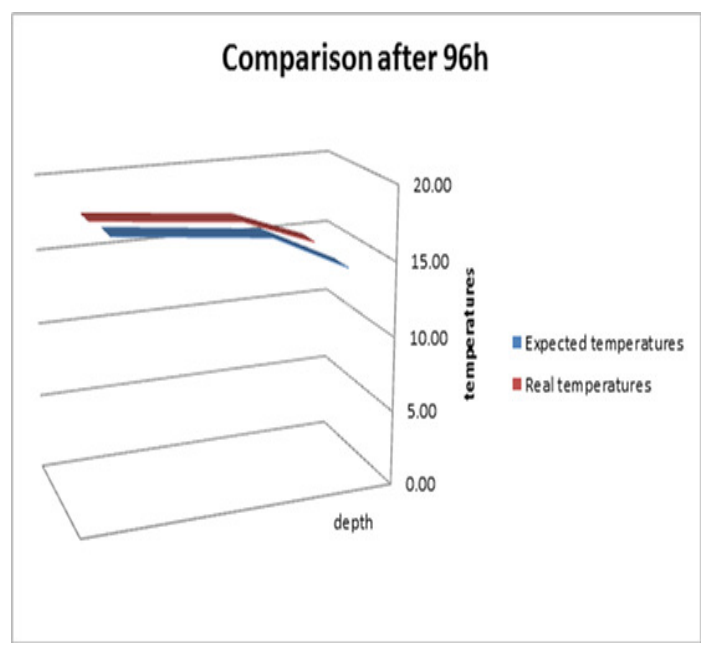

Figure 18: Comparison after 96h. 


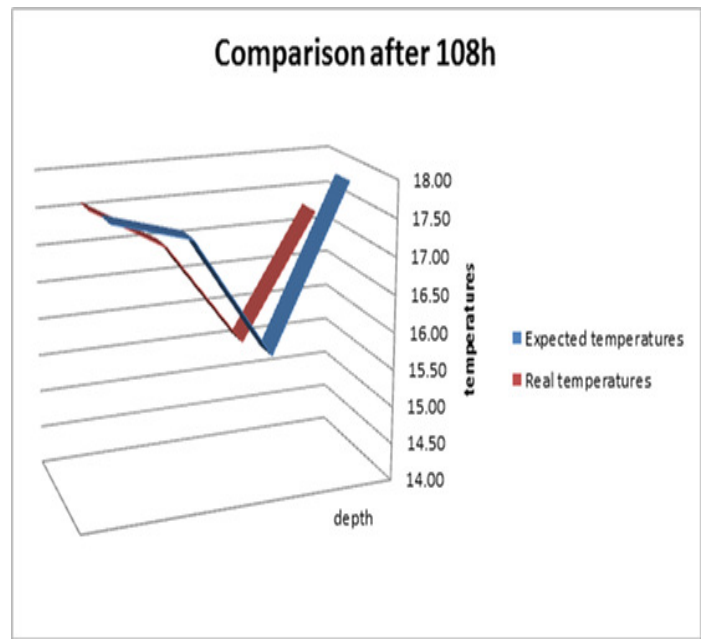

Figure 19: Comparison after 108h. 